\title{
Syphilis in Blood Donors in Tertiary Care Teaching Hospital
}

\author{
Ravi Jain And Ashok Yadav* \\ Dept. of Pathology, MGM Medical College, Indore, India
}

\begin{abstract}
Introduction: Transmission of infectious diseases through donated blood is of concern to blood safety as transfusion forms an integral part of medical and surgical therapy. Blood transfusion carries the risk of transfusion-transmissible infections, including HIV, hepatitis, syphilis, malaria and infrequently toxoplasmosis.
\end{abstract}

Aims \& Objectives: To find out the seroprevalence of Syphilis in blood donors, to find the incidence of spectrum of Transfusion transmitted dieasess in blood bank donation \& to find the age distribution of the cases studied.

Material \& Methods: The present study was undertaken in the Department of Pathology MGM Medical College Indore. This is a retrospective study that was conducted, during the period 2008 -2015. The screening for Syphilis was done by rapid chromatographic assay for detection of antibodies to T. pallidum

Results: Out of total 137689 blood donations, majority of donors are voluntary donors $83.02 \%$ as compared to replacement donors $17.05 \%$. Seroprevalence of Anti TP is $0.26 \%$. Seroprevalence of Anti TP is higher in the age group 26-35 year. Among Voluntary \& replacement/relative donors. Overall seropositivity of TTI's (HIV, HBV, HCV, Syphilis \& Malaria) is higher in replacement donors $3.71 \%$ as compared to voluntary donors $1.75 \%$.

Conclusion: voluntary blood donation should be encouraged for prevention of transfusion-transmissible diseases. The time and cost involved in screening donated blood can be reduced by an effective donor education and selection program that promotes self-exclusion by donors at risk of transfusion-transmissible infections.

Keywords: Treponema Pallidum, Seroprevalence, Transfusion Transmitted Diseases, Voluntary Donors, Replacement Donors

\section{Introduction}

Transmission of infectious diseases through donated blood is of concern to blood safety as transfusion forms an integral part of medical and surgical therapy. Blood transfusion carries the risk of transfusion-transmissible infections, including HIV, hepatitis, syphilis, malaria and infrequently toxoplasmosis, Brucellosis and some viral infections like CMV, EBV and herpes.

With every unit of blood, there is $1 \%$ chance of transfusionassociated problems including transfusion-transmitted diseases.Among all infections HIV and hepatitis are the most dreadful. The first case of transfusion-associated AIDS was described in an infant given transfusion for erythroblastosis foetalis. Thereafter, many cases were reported all over the world in which transfusion of blood and its products was the only risk factor.The improved screening and testing of blood donors has significantly reduced transfusion-transmitted diseases in most developed countries. This has not been so in developing nations. Poor health education and lack of awareness result in the reservoir of infections in the population.

There are four main groups of micro-organisms known to cause infections namely viruses, bacteria, protozoa and fungi. Only first three groups of microbes - viruses, bacteria + spirochetes and protozoa - have been reported to be transmitted by blood transfusion. Individuals with fungal infections are usually too sick to be accepted as blood donors. Viruses are most commonly transmitted by transfusion.

Recently, a new form of infectious agent - the prion - has been identified. At this time, there is no evidence to suggest that they could be transmitted by blood transfusion.

Viruses are the simplest forms of life. They infect all forms of life, they lack certain components needed to live and their growth hence depend on the host cell that they infect to provide these missing components.Following are some of the viruses which are known to be transmitted through blood:

1. Human immunodeficiency virus (HIV)

2. Hepatitis $B$ virus

3. Hepatitis $\mathrm{C}$ virus

4. Hepatitis A virus

5. Hepatitis $\mathrm{G}$ virus

6 Non - A, Non - B Hepatitis 
7. Epstein Barr Virus

8. Cytomegalo virus (CMV)

9. Human T Lymphocytic virus (HTLV - 1 \& HTLV - 2)

Syphilis, an ancient disease is caused by Spirochete Treponema pallidum. According to The World Health organization estimates ${ }^{2}$, there are approximate 12 million new cases diagnosed each year, with more than $90 \%$ in developing countries. Syphilis has acquired new potential for morbidity with the advent of HIV \& AIDS.

Natural History of syphilis ${ }^{3}$ : Syphilis is a chronic disease caused by Treponema pallidum. Treponemes (trepos to turn \& nema - thread) ${ }^{4}$ are relatively short, slender spirochetes with fine spirals \& pointed or round ends. Some of them are pathogenic while others occur as commensals in the mouth, intestines \& genitalia. Treponemes causes the following diseases in the humans ${ }^{4}$.

1. Venereal Syphilis: Treponema pallidum.

2. Endemic Syphilis: Treponema pallidum (Tendemicum)

3. Yaws: Treponema pertenue

4. Pinta: Treponema carateum

They are almost identical in their morphology \& antigenic structure though there are differences in clinical features \& natural history.

Treponema Pallidum: the causative agent of syphilis was discovered by Schaudinn \& Hoffmann $(1905)^{4}$ in the chancres \& inguinal lumph nodes of syphilitic patients. The name pallidum refers to its pale staining. Treponema pallidum is a thin, delicate spirochete with tapering ends, about $10 \mu \mathrm{m}$ long (range 4-14 $\mu \mathrm{m}$ ) \& 0.1-0.2 $\mu \mathrm{m}$ wide. It has ten regular spirals, which bare sharp, angular at regular intervals. The spirochete is actively motile, rotating around long axis with backward \& forward movements. Treponema pallidum cannot be seen under light microscope but can be appreciated under dark ground or phase contrast microscope. Treponema pallidum can be demonstrated in tissue sections using silver impregnation methods ${ }^{4}$.

Antigenic Structure of Treponema Pallidum: Infection induces three types of antibodies ${ }^{4}$.

1. Reagin Antibody: reacts in Wasswrmann, Kahn \& VDRL tests, in which hapten extracted from beef heart (Cardiolipin) is used as Antigen.

2. Group Antigen: found in Treponema pallidum \& non-pathogenic treponemes.

3. Species Specific: antibody to this antigen is demonstrated by specific Treponema pallidum tests $\&$ which are positive in sera of patients infected with Treponema pallidum.
On the basis of clinical presentation, infectivity \& progression Syphilis is divided into 5 clinical stages primary, secondary, latent \& tertiary syphilis.

1. Primary Syphilis: painless genital ulcer (chancre) following exposure to infection.

2. Secondary Syphilisl macular popular rash involving palms \& soles with high bacteriemia in blood.

3. Latent Phase: asymptomaticbut positive serological test for syphilis

4. Tertiary Syphilis: cardiovascular/neurological symptoms.

Transfusion Transmitted Syphilis: The first case of Transfusion transmitted syphilis was reported in $1915^{3}$. 138 cases were reported in the literature by $1941^{5}$. Cases were mostly discovered in donors with primary or secondary stage of disease ${ }^{6}$. Treponema pallidum may be found in blood, not levels are variable \& bacteremia is short lived. Treponemes are senisitve to cold; hence risk of transmission through stored blood at $4-8^{\circ} \mathrm{C}$ is very $10 w^{7-8}$.

In India, most blood donors are first-time donors ${ }^{3}$. The prevalence of syphilis among blood donors in India was reported to be $0.7 \%{ }^{9}$. The global incidence of syphilis in blood donors is variable ranging from $0.75 \%$ in Pakistan ${ }^{10}$ to $12.7 \%$ in Tanzania ${ }^{11}$.

Testing Methodologies ${ }^{3}$ : Three methods are currently used.

1. Direct microscopic examination in early stage.

2. Non-treponemal Serological Tests e.g : RPR \& VDRL

3. Indirect Treponemal Tests: FTA-ABS(Fluorescent treponemal antibody absorption test), TPHA (Treponema pallidum Hemagluttination assay), EIA (enzyme immunoassay), Rapid assays (immunechromatographic strips) \& western Blotting

4. Molecular Based Methods: PCR (Polymerase chain reaction)

\section{Aims and Objectives}

The study is being conducted in the department of pathology, M.G.M.M.C, Indore.

1. To find out the seroprevalence of Syphilis in blood donors.

2. To find the age distribution of the cases studied.

3. To find the incidence of spectrum of Transfusion transmitted diseases in blood bank donation.

Material and Methods

The present study is being undertaken in the Department of Pathology MGM Medical College Indore. This is a 
retrospective study that will be conducted, during the period $2008-2015$. Tests are routinely done on every blood unit to exclude HIV, HBV, HCV, syphilis and malaria. Donors were selected by the standard criteria for donor fitness. The screening for Syphilis was done by rapid chromatographic assay for detection of antibodies to T.pallidum . ABO and Rhesus (Rh) blood groups were determined using blood grouping antisera: anti-A, anti-B, anti-AB, and anti-D. Selection of cases for the study included the donors of MYH Blood Bank.

\section{Results}

The present study was conducted in the Department of Pathology MGM Medical College Indore and M. Y. Hospital blood bank. This is a retrospective study that was conducted, during the period 2008 -2015. In the present study, 137689 blood donors are observed in the year 200815 in the M. Y. Blood Bank. The data collected from donor register record book, donors form, master record book, HIV, \& HBV positive bag number records. The results and observations studies are presented below:
Graph 1: Out of total 137689 blood donations, majority of donors are voluntary donors $83.02 \%$ as compared to replacement/relative donors $17.05 \%$

Graph 2: Out of total 137689 blood donations, majority of donors are male donors $95.59 \%$ as compared to female donors $4.40 \%$

Graph 3: Seropositive donors for Anti TP in 2008-15. Seroprevalence of Anti TP is $0.26 \%$.

Graph 4: Age wise distribution of Anti TP in the year 2008-15. Seroprevalence of Anti TP is higher in the age group 26-35 year

Table 1: Seropositivity of transfusion transmitted diseases (HIV, HBV, HCV, Syphilis \& Malaria) in 200815. Among Voluntary \& replacement/relative donors . Overall seropositivity of TTI's (HIV, HBV, HCV, Syphilis $\&$ Malaria) is higher in replacement donors $3.71 \%$ as compared to voluntary donors $1.75 \%$.

Table 1: Seropositivity of transfusion transmitted diseases (HIV,HBV,HCV,Syphilis \& Malaria) in 2008-15. Among Voluntary \& replacement/relative donors . Overall seropositivity of TTI's (HIV,HBV,HCV,Syphilis \& Malaria) is higher in replacement donors $3.71 \%$ as compared to voluntary donors $1.75 \%$.

\begin{tabular}{|l|l|l|l|l|} 
S.No & $\begin{array}{l}\text { Total No of } \\
\text { Voluntary donors } \\
(\mathbf{2 0 0 8 - 1 5 )}\end{array}$ & $\begin{array}{l}\text { Total No of Voluntary } \\
\text { donors found } \\
\text { seropositive for TTI } \\
\mathbf{( 2 0 0 8 - 1 5 )}\end{array}$ & $\begin{array}{l}\text { Total No of } \\
\text { Replacement/ } \\
\text { relative donors } \\
(\mathbf{2 0 0 8 - 1 5 )}\end{array}$ & $\begin{array}{l}\text { Total No of } \\
\text { Replacement/relative } \\
\text { found seropositive for } \\
\text { TTI (2008-15) }\end{array}$ \\
\hline Number & 114246 & 2007 & 24093 & 896 \\
\hline \%age & $83.02 \%$ & $1.75 \%$ & $17.05 \%$ & $3.71 \%$ \\
\hline
\end{tabular}

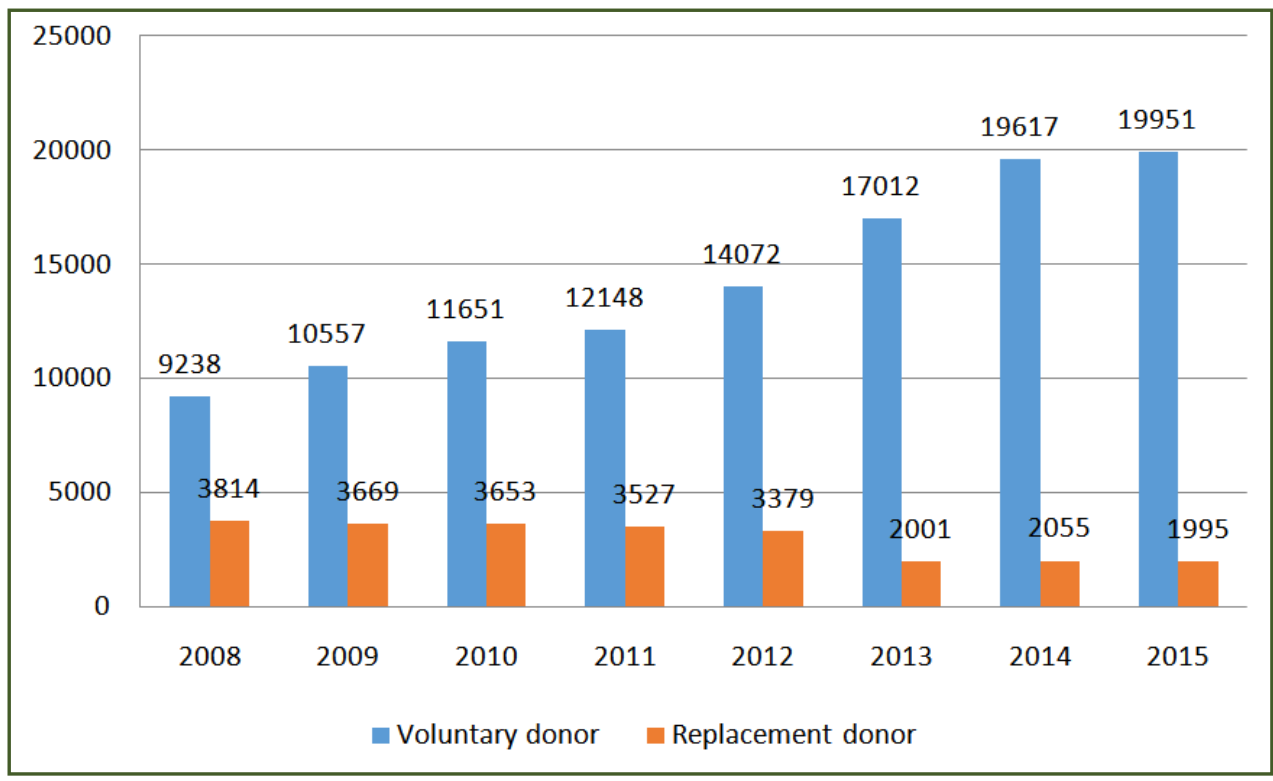

Graph 1: Number of blood units collected during the year 2008-15. Out of total 137689 blood donations, majority of donors are voluntary donors $\mathbf{8 3 . 0 2} \%$ as compared to replacement/relative donors $\mathbf{1 7 . 0 5} \%$ 


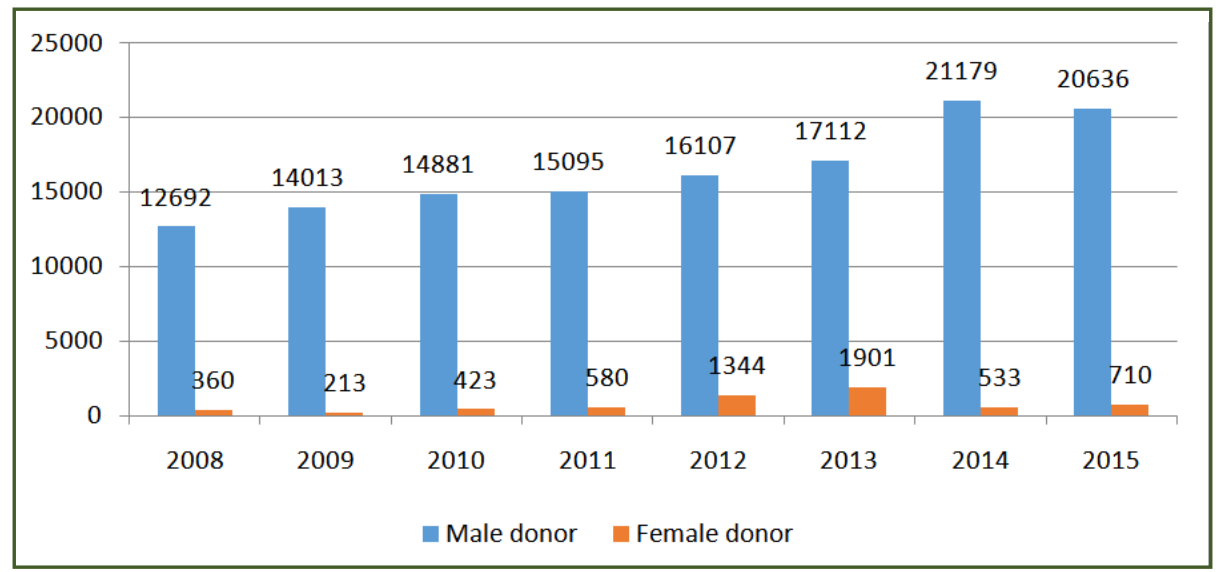

Graph 2 : Number of male and female donors during the year 2008-15.0ut of total 137689 blood donations, majority of donors are male donors $95.59 \%$ as compared to female donors $4.40 \%$.

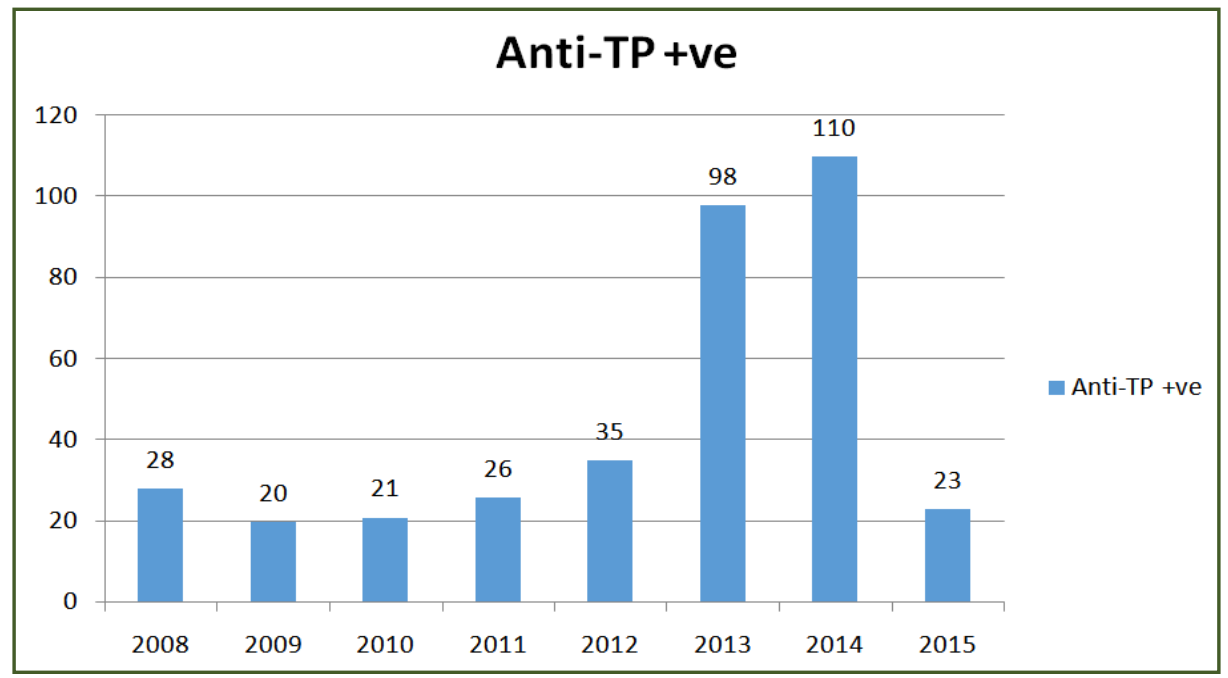

Graph 3 : Seropositive donors for Anti TP in 2008-15 Seroprevalence of Anti TP is $0.26 \%$.

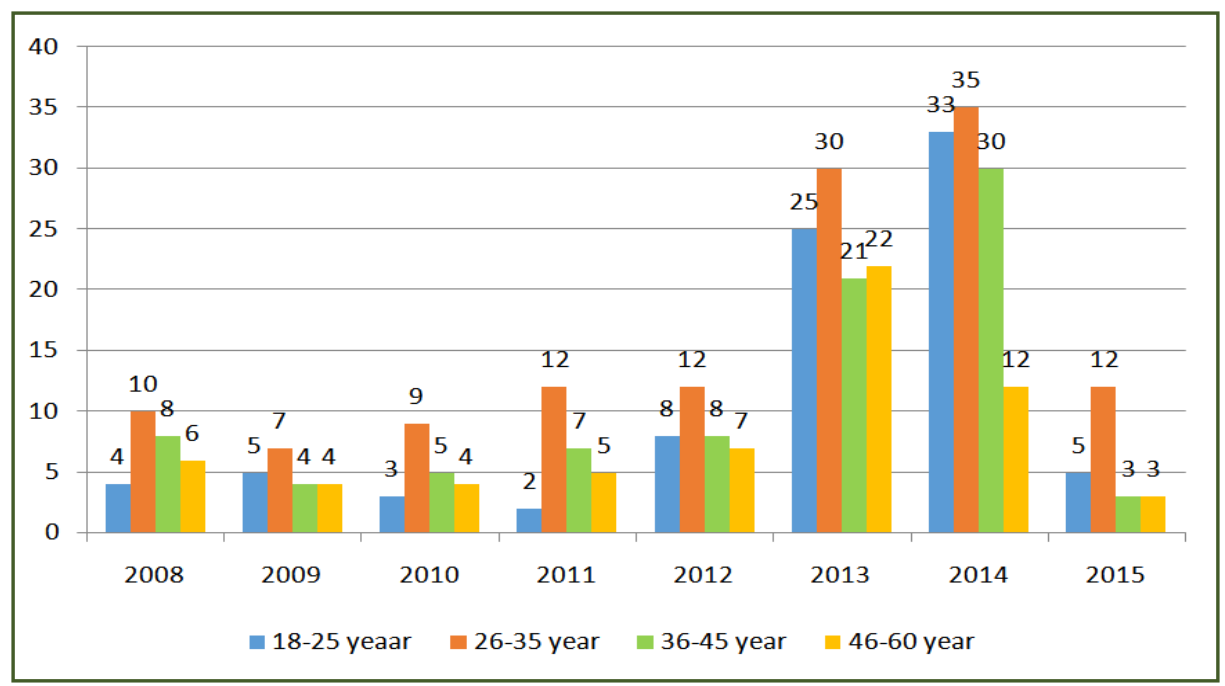

Graph 4: Age wise distribution of Anti TP in the year 2008-15. Seroprevalence is higher in the age group 26-35 year

http://www.pacificejournals.com/aabs 


\section{Discussion}

Voluntary or Replacement/Relative Donor -In our study, out of total 137689 blood donations, majority of donors are voluntary donors $83.02 \%$ as compared to replacement/ relative donors $17.05 \%$ (Graph 1) Similarly majority of donors are voluntary in another study by Nagarekha Kulkarni $^{12}$, „, out of 19135 blood donors, 11165 (58\%) were voluntary and 7970 (42\%) were replacement donors

Male or Female Donors: In our study, out of total 137689 blood donations, majority of donors are male donors $95.59 \%$ as compared to female donors $4.40 \%$ (Graph 2) . Similarly another study is comparable for majority of donors are male $96.22 \%$ by Dimple Arora and Bharti Arora et $\mathrm{al}^{13}$ in Haryana. In the another study, the percentage of male patients was $73 \%(860 / 1178)$ as compared with $27 \%$ (318/1178) for female patients by Manisha Jain et al ${ }^{14}$, conducted in New Delhi.

Seroprevalence of Anti TP (Anti Treponema pallidum): In our study, the Seroprevalence of Anti TP is $0.26 \%$. in total blood donations in the year 2008-15 (Graph 3). Seroprevalence of Syphilis is comparable to another study with seroprevalence of Anti TP was $0.91 \%$ by Sultan S, Murad S Irfan $\mathrm{m}$ et al ${ }^{15}$ conducted in Pakistan. In another study by Elyamany $\mathrm{G}$ et $\mathrm{al}^{16}$ seroprevalence was found to be $0.044 \%$. In a study conducted at Mangalore, India by Zulfikar A et al ${ }^{17}$ seropositivity of syphilis was found to be $0.07 \%$.

Age Wise Distribution: In our study, overall Seroprevalence of Anti TP (2008-15) is higher in the age group 26-35 years for anti TP (0.09\%) (Graph 4 ). In a study conducted at Mangalore, India by Zulfikar A et al ${ }^{17}$ incidence of seropositivity was found to be more in donors in the group aged 18-35 years old than in the group 36-55 years old. In a study by Tessema et al ${ }^{18}$, seropositivity of syphilis was found to be $0.9 \% \& 1.7 \%$ in age groups $17-25$ \& 26-35 yrs respectively. In another study by Elyamany $\mathrm{G}$ et $\mathrm{al}^{16}$ seropositivity was found to be highest in age group 21-30 yrs.

Seropositivity in Volunatry/replacement Donors: Among Voluntary \& replacement/relative donors, Overall seropositivity of TTI's (HIV, HBV, HCV, Syphilis \& Malaria) is higher in replacement donors 3.71 $\%$ as compared to voluntary donors $1.75 \%$ (Table 1 ). In study by Nagarekha Kulkarni ${ }^{12}$, the seroprevalence was more in relative/replacement donors as compared to voluntary donors.

\section{Conclusion}

The present study was conducted in the Department of Pathology MGM Medical College Indore and M. Y.
Hospital blood bank. This is a retrospective study that was conducted, during the period $2008-2015$. Tests are routinely done on every blood unit to exclude HIV, HBV and HCV. Donors were selected by the standard criteria for donor fitness. The data collected from donor register record book, donors form, master record book, HIV, HBV and HCV positive beg number records. Out of total 137689 blood donations, majority of donors are voluntary donors $83.02 \%$ as compared to replacement/relative donors $17.05 \%$. Out of total 137689 blood donations, majority of donors are voluntary donors $83.02 \%$ as compared to replacement donors $17.05 \%$. Seroprevalence of Anti TP is $0.26 \%$. Seroprevalence of Anti TP is higher in the age group 26-35 year. Among Voluntary $\&$ replacement/relative donors . Overall seropositivity of TTI's (HIV,HBV,HCV,Syphilis \& Malaria) is higher in replacement donors $3.71 \%$ as compared to voluntary donors $1.75 \%$. HBV and HIV are the most prevalent transfusion-transmissible diseases among blood donors in Indore. Screening and better selection of donors are necessary to improve blood safety in the regional blood transfusion centre of M. Y. Hospital. Therefore, it is concluded that voluntary blood donation should be encouraged for prevention of transfusion-transmissible diseases. The time and cost involved in screening donated blood can be reduced by an effective donor education and selection program that promotes self-exclusion by donors at risk of transfusion-transmissible infections.

\section{Acknowledgements}

We are Highly grateful to Dr C.V.Kulkarni, Prof.\& Head, Dept. of Pathology for providing us the opportunity \& full support.

\section{References}

1. Tang, J. and Kaslow, R. A. (2003). "The impact of host genetics on HIV infection and disease progression in the era of highly active antiretroviral therapy". AIDS 17 (Suppl 4): S51-S60.

2. World Health Organization. Global prevalence \& incidence of selected curable sexually transmitted infections: overview \& estimates (2001). Available at : http://www.who.int/hiv/ pub/sti/en/who_hiv_aids_2014.05.pdf.

3. Kaur.G, Kaur.P : Syphilis testing in blood donors: an update. Blood Transfus 2015;13:197-204

4. Ananthnarayan.R \& Panicker C.K in Ananthnarayan. \& Panicker's Textbook of Microbiology 8th Edition Chapter 42 pg 371. University Press.

5. De Schryver A, Meheus A. Syphilis \& blood transfusion: a global perspective. Transfusion 1990;30:844-7

6. Gardella C, Marin AA, Kahn RH et al. Persons with early syphilis identified through blood or plasma donation screening in United states. J Infect Dis 2002; 185:545-9 
7. Orton S. Syphilis \& blood donors: what we know, what we do not know, \& what we need to know. Transfus Med Rev 2001;15:282-91.

8. Wendel S. Current concepts on transmission of bacteria \& parasites by blood components. Vox Sang 1994;67(Supp13):161-74

9. Kaur G, Basu S, Kaur R et al. Patterns of infections blood donors in a tertiary care center: A retrospective study. Natl Med J India 2010:23;147-9

10. Bhalti FA, Ullah Z, Salamat N, et al . Anti-Hepatitis B core antigen testing, viral markers \& occult Hepatitis b infection in Pakistani blood donors: implication for transfusion practice. Transfusion 2007; 47:74-9

11. Matee MI, Magesa PM, Lyamuya EF. Seroprevalence of Human immunodeficiency virus, Hepatitis B \& C virus and syphilis infections among blood donors at Muhimbili National hospital in Dar es Salam, Tanzania. BMC Public health 2006;6:21

12. Kulkarni N. Analysis of the seroprevalence of HIV, HBsAg, $\mathrm{HCV}$ and syphilitic infections detected in the pretranfusion blood: A short report. International Journal of Blood Transfusion and Immunohematology 2012;2:1-3.
13. Dimple Arora, Bharti Arora, AnshulKhetarpal"Seroprevalence of HIV, HBV, HCV and syphilis in blood donors in Southern Haryana" Year 2010 Vol: 53(2)Page308-309

14. Manisha Jain, Anita Chakravarti, VikasVerma, PreenaBhalla"Seroprevalence of hepatitis viruses in patients infected with the human immunodeficiency virus" Year : 2009 Vol: 52( 1)Page : 17-19

15. Sultan S, Murad S Irfan $\mathrm{m}$ et al . Trends of venereal infections among healthy blood donors at Karachi. Arch Iran Med 2016;19(3):192-196

16. Elyamany $\mathrm{G}$ et al. Prevalence of syphilis among blood \& stem cell donors in Saudi Arabia: an institutional experience.2016; Vol 8 Issue 8: 2747-2751

17. Zulfikar A, Umaru N, Shreesha K. Seroprevalence of Transfusion transmitted Infections among blood donors in Mangalore. Medica Innov. Dec 2012, Vol 1, issue 2; 24-27

18. Belay Tessema et al "Seroprevalence of HIV, HBV, HCV and syphilis infections among blood donors at Gondar University Teaching Hospital, Northwest Ethiopia: declining trends over a period of five years" BMC Infectious Diseases201010:111

*Corresponding author:

Dr. Ashok Yadav, Postal Address: Yadav clinic, Newpanchsheel colony, Musakhedi, Indore (M.P )452001

Phone: +91 9893273236

Email: drashokmyh@gmail.com,ravijainpatho@gmail.com 